\begin{tabular}{|c|l|}
\hline Title & Flow rate driven by peristaltic movement in plasmodial tube of Physarum polycephalum \\
\hline Author(s) & Y amada, Hiroyasu; Nakagaki, Toshiyuki \\
\hline Citation & $\begin{array}{l}\text { A merican Institute of Physics Conference Proceedings, 1028, 210-214 } \\
\text { https://doi.org/10.1063/1.2965090 }\end{array}$ \\
\hline Issue Date & 2008-07-03 \\
\hline Doc URL & http://hdl.handle.net/2115/34764 \\
\hline Rights & $\begin{array}{l}\text { Copyright 2008 A merican Institute of Physics. This article may be downloaded for personal use only. A ny other use } \\
\text { requires prior permission of the author and the A merican Institute of Physics. }\end{array}$ \\
\hline Type & article (author version) \\
\hline File Information & Yamada_Nakagaki.pdf \\
\hline
\end{tabular}

Instructions for use 


\title{
Flow rate driven by peristaltic movement in plasmodial tube of Physarum polycephalum
}

\author{
Hiroyasu Yamada* and Toshiyuki Nakagaki ${ }^{\dagger}$ \\ *Harukidai 5-1-2, Togo, Aichi 470-0161, Japan, and \\ Bio-Mimetic Control Research Center, \\ The Institute of Physical and Chemical Research (RIKEN), \\ Nagoya 463-0003, Japan \\ ${ }^{\dagger}$ Research Institute for Electronic Science, \\ Hokkaido University, Sapporo, 060-0812, Japan, and \\ Creative Research Initiative "SOUSEI", \\ Hokkaido University, Sapporo, 001-0021, Japan.
}

\begin{abstract}
We report a theoretical analysis of protoplasmic streaming driven by peristaltic movement in an elastic tube of an amoeba-like organism. The plasmodium of Physarum polycephalum, a true slime mold, is a large amoeboid organism that adopts a sheet-like form with a tubular network. The network extends throughout the plasmodium and enables the transport and circulation of chemical signals and nutrients. This tubular flow is driven by periodically propagating waves of active contraction of the tube cortex, a process known as peristaltic movement. We derive the relationship between the phase velocity of the contraction wave and the flow rate, and we discuss the physiological implications of this relationship.
\end{abstract}

Keywords: Physarum, peristaltic movement, fluid mechanics, circulation

PACS: 47.70.Fw, 87.17.Aa, 47.63.-b

\section{INTRODUCTION}

The body of the plasmodium of Physarum polycephalum consists of a network of tubular elements by means of which nutrients, chemical signals and protoplasm circulate through the organism. The motive force of this streaming is hydrodynamic pressure, which is produced by the propagation of waves of contraction activity along a tube wall. This process is known as peristaltic movement and it allows several important functions to take place in the plasmodium: 1) the organism can migrate because body mass is transported by the peristaltic movement; 2) chemical signals can be communicated through the tubular network; 3) nutrients can be delivered throughout the organism. Here we report a hydrodynamical analysis of the flow rate that results from peristaltic movement. The theoretical results obtained are compared with the behavior observed experimentally in the plasmodium.

\section{BASIC EQUATIONS FOR PERISTALTIC MOVEMENT AND APPROXIMATIONS FOR PLASMODIAL FLOW}

We consider a flow of incompressible fluid in a circular, cylindrical, elastic tube of infinite length. For the sake of simplicity, we assume that the thickness of the tube wall 
is zero. We begin with the law of conservation of mass and momentum, as found in standard textbooks[1]. When $A(x, t)$ is the cross-sectional area of the tube and $u(x, t)$ is the mean velocity of flow over the cross-section of the tube, both of which are functions of the axial coordinate $x$ and time $t$, then the conservation of mass is given by

$$
\frac{\partial A}{\partial t}+\frac{\partial}{\partial x}(u A)=0
$$

When $p(x, t)$ is the pressure of the fluid inside the tube, the conservation of momentum is expressed as

$$
\frac{\partial u}{\partial t}+u \frac{\partial u}{\partial x}=-\frac{1}{\rho} \frac{\partial p}{\partial x}-R u,
$$

where $\rho$ is the (constant) density of the fluid and $R$ is the friction coefficient of the fluid in contact with the tube wall. For simplicity, we assume that $R$ is constant; however, in general it is a function of $A$.

Next, we consider $p(x, t)$, which is a function of $A$. We assume the relationship

$$
p-p_{e x}=f(A)
$$

where $p_{e x}$ is the external pressure that gives rise to peristaltic movement. The function $f$ represents the elasticity of the tube when it expands and contracts laterally, and is a monotone increasing function of $A$. Equation 3 implies that the dynamics between $p_{\text {ex }}$ and $A$ is replaced by an equation of statics and that the mass of the tube wall is negligible. We note that equations 1 - 3 were originally defined in a previous study of blood vessels [1].

In order to describe the peristaltic movement in Physarum, we express the external pressure $p_{e x}$ as

$$
p_{e x}=p_{0} e^{i(k x-\omega t)} .
$$

Equations 1 - 4 are then written in the vector form

$$
\frac{\partial}{\partial t}\left[\begin{array}{l}
A \\
u
\end{array}\right]+\left[\begin{array}{cc}
u & A \\
f^{\prime} / \rho & u
\end{array}\right] \frac{\partial}{\partial x}\left[\begin{array}{c}
A \\
u
\end{array}\right]=\left[\begin{array}{c}
0 \\
-(1 / \rho) \partial p_{e x} / \partial x-R u
\end{array}\right]
$$

where the first and second terms on the right-hand side are the injected momentum and the momentum dissipated by friction, respectively. These equations are of the hyperbolic type. The coefficient matrix on the left-hand side contains the eigenvalues $\lambda_{ \pm}=u \pm c$, which correspond to forward and backward waves, where $c$ is the sonic speed of the elastic tube, $c=\sqrt{A f^{\prime} / \rho}$. We rescale the equations by the dimensionless parameters,

$$
\tau=k c_{0} t, \xi=k x, \alpha=\frac{A}{A_{0}}, \nu=\frac{u}{c_{0}}, \Pi=\frac{p}{\rho c_{0}^{2}},
$$

where $c_{0}$ is the sonic speed for the mean cross-sectional area; $c$ is a function of the crosssectional area and is given by $c_{0}=\sqrt{A_{0} f^{\prime}\left(A_{0}\right) / \rho}$. As above, the sonic speed is chosen 
as the rescaling factor. We then obtain

$$
\begin{array}{r}
\frac{\partial \alpha}{\partial \tau}+\frac{\partial}{\partial \xi}(v \alpha)=0, \\
\frac{\partial v}{\partial \tau}+\frac{\partial v}{\partial \xi}=-\frac{\partial \Pi}{\partial \xi}-\gamma v,
\end{array}
$$

where

$$
\begin{array}{r}
\Pi=F(\alpha)+\Pi_{0} e^{i(\xi-\mu \tau)}, F(\alpha)=\frac{f\left(A_{0}\right) \alpha}{A_{0} f^{\prime}\left(A_{0}\right)}, \\
\Pi_{0}=\frac{p_{0}}{\rho c_{0}^{2}}, \mu=\frac{\omega / k}{c_{0}}, \gamma=\frac{R}{k c_{0}} .
\end{array}
$$

In this dimensionless system, the sonic speed is normalized because $\sqrt{F^{\prime}(1)}=1$ for the normalized cross-sectional area, $\alpha=1$. The parameter $\mu$ is important because it expresses the ratio of the sonic speed to the wave speed of the peristaltic movement. In the actual organism, we can assume that $\omega$ is nearly constant. We solve this system of equations numerically.

\section{SIMULATION RESULTS}

Figure 1 shows the relationship between pressure and flux for different values of $\mu$ and $\gamma$ when $\frac{d F(\alpha)}{d \alpha}$ is a non-zero constant (stress is proportional to the cross-section). When a contraction wave passes through a tube, the pressure varies from maximum to minimum in cyclical fashion. The flux also changes with the pressure. Thus, the relationship between pressure and flux takes the form of a closed curve, as shown in Fig.1. The flux reaches its maximum and minimum values close to where pressure $=1$ because the pressure gradient of the sinusoidal wave is maximum and minimum at these points, respectively. The flux is zero close to the maximum and minimum pressures because the gradient is zero here. The flow direction is both negative and positive during the course of a cycle, but the net flux is positive with respect to the propagation direction of the contraction wave. In general, the pressure-flux curves shrink as the wave propagation becomes slower ( $\mu$ is smaller). Therefore, more protoplasmic sol moves back and forth during a flow cycle when the wave propagates faster. This implies that communication via chemical signals can occur more densely, because the rate of exchange of chemical material between neighboring elements along a tube increases.

However, it is not trivial to establish whether the net flux is also a function of the wave propagation speed. Figure 2 shows the net flux per peristaltic cycle and per unit time, both as a function of peristaltic speed. The plot of net flux per cycle is bell-shaped and the flux reaches an optimum value at a finite peristaltic speed before decreasing again. The net flux per unit time is approximately proportional to the peristaltic speed when the peristaltic speed is small, but reaches a constant value at higher speeds. 


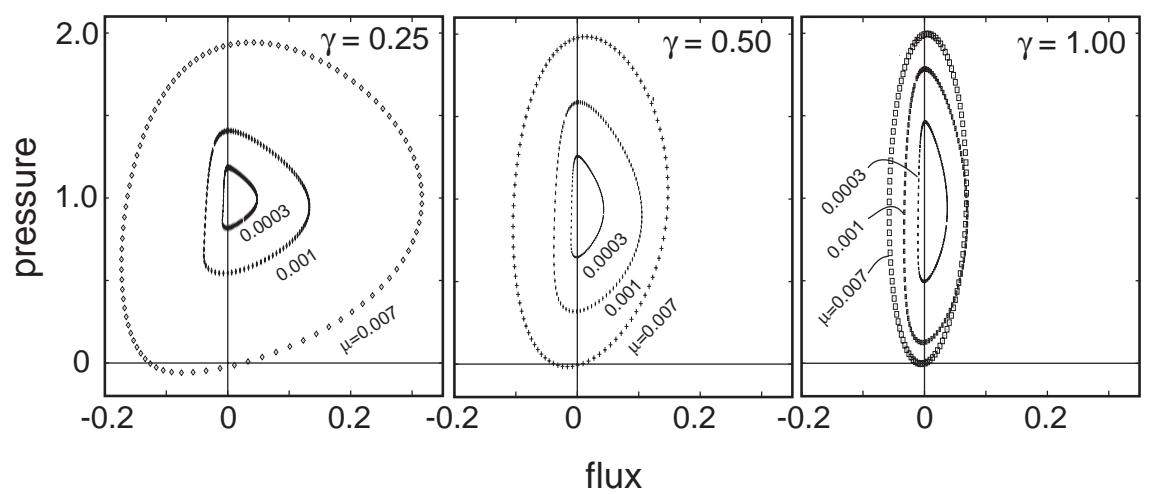

FIGURE 1. Flux-pressure relationship for different values of $\gamma$ and $\mu$. Left: $\gamma=0.25$. Center: $\gamma=0.50$. Right: $\gamma=1.00$. In each panel, plots for $\mu=0.0003$ (small loop), 0.0010 (medium loop) and 0.0070 (large loop) are shown. In all cases, $F(\alpha)=\alpha$ and $\Pi_{0}=1.0$.
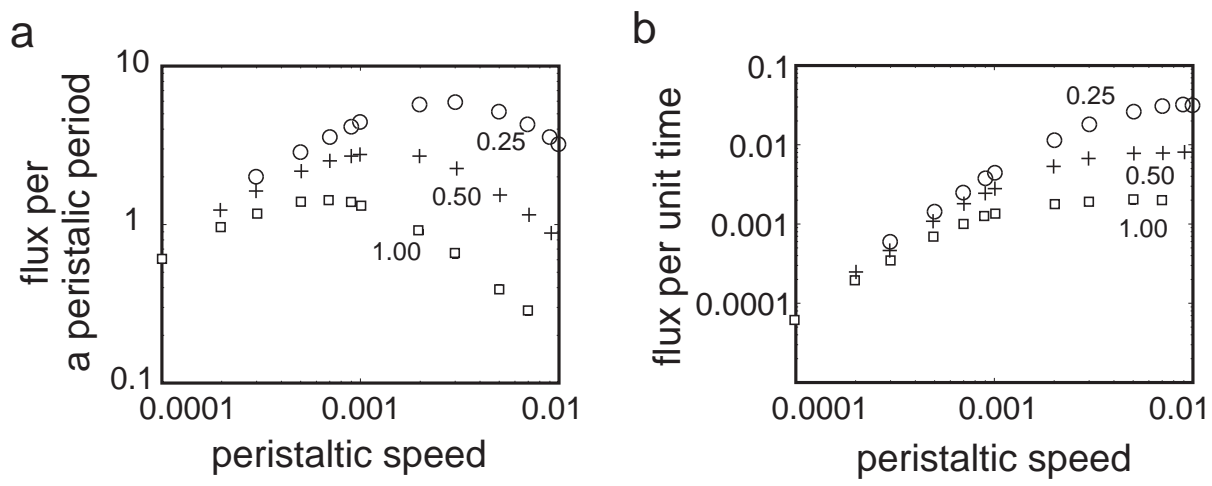

FIGURE 2. Flow rate as a function of peristaltic speed: (a) Flux per peristaltic period; (b) Flux per unit time. Plots are shown for $\gamma=0.25,0.50$ and 1.00. In all cases, $F(\alpha)=\alpha$ and $\Pi_{0}=1.0$.

\section{ESTIMATION OF PERISTALTIC SPEED IN PLASMODIUM}

Here we estimate the peristaltic speed $\mu$ in the plasmodium, where $\mu=(\omega / k) /\left(c_{0}\right)$ and $c_{0}=\sqrt{A_{0} f^{\prime}\left(A_{0}\right) / \rho}$. The parameter $\mu$ is approximately $2 \times 10^{-4}$ for the following experimentally measured values: $\omega=10^{-2} \mathrm{sec}^{-1}, k=10^{-1} \mathrm{~m}^{-1}, \Delta P=5 \times 10^{3} \mathrm{~Pa}$, $\Delta A=10^{-7} \mathrm{~m}^{2}, A_{0}=8 \times 10^{-7} \mathrm{~m}^{2}$ and $\rho=10^{3} \mathrm{~kg} \mathrm{~m}^{-3}$. We assume that the function $f(A)$ is proportional to the cross-section $A$ and is obtained as $(\Delta P) /(\Delta A)$. As the size of the plasmodium increases from $1 \mathrm{~cm}$ to $100 \mathrm{~cm}, \mu$ increases from $2 \times 10^{-5}$ to $2 \times 10^{-3}$. These $\mu$ values are near the optimum values of flux per peristaltic cycle and are also near the saturation values of flux per unit time. This implies that the actual peristaltic speed in the organism is effective for the transportation of protoplasmic sol. According to this analysis, we can conclude that the flow rate is greater in a large plasmodium $(100 \mathrm{~cm})$ than in a small one (less than $1 \mathrm{~cm}$ ), which demonstrates the merit of large systems. 


\section{DISCUSSION}

We now address the physiological implications of the flow rate. The maximum flow rate per peristaltic period, at the top of the bell-shaped curve in Fig. 2a, corresponded to an optimum flow speed of $\left(\mu_{1}^{\star}\right)$. The flow rate per unit time increased with respect to flow speed and saturated at the flow speed $\mu_{2}^{\star}$ (Fig. 2b). The values of $\mu_{1}^{\star}$ and $\mu_{2}^{\star}$ were similar, and we thus denote them by $\mu^{\star}$. The flow rate $\mu^{\star}$ is convenient for physiological purposes because the power increases as the flow speed increases. A balance must be reached between the cost of achieving a high power and the benefit of obtaining a high net flow rate. Therefore, it is optimal for the organism that the benefit is maximized at minimum cost.

The measured flow speed of the plasmodium was less than $\mu^{\star}$, and thus the flow rate did not reach the maximum possible value. For example, the flow rate was $2 \times 10^{-5}$ for the plasmodium of diameter $1 \mathrm{~cm}$. The flow speed increased as the plasmodium became larger and became close to the optimum value of $\mu^{\star}$ for the plasmodium of 100 $\mathrm{cm}$ diameter. Therefore, increasing the size of the organism to the order of one meter is beneficial because the flow rate can be optimized. This may be a reason why the plasmodium can extend to such large dimensions. This effective flow enables chemical and physical signals to be transported efficiently throughout the organism.

\section{REFERENCES}

1. Y. C. Fung, Biomechanics -Circulation- (2nd edition), Springer-verlag, New York, 1997, pp. 140-143. 